\title{
Stage classification and prognosis: an intersection of medicine, quantum physics and religion?
}

\section{Frank C Detterbeck}

Estimating prognosis is an important part of caring for patients with cancer. However, predicting prognosis is complicated and depends on many factors. Simply amassing more data alone is not the answer; we have to learn to intellectually manage the inherent complexity and uncertainty if we are to make progress.

The 7th edition of the Cancer Stage Classification System resulted in moving several lung cancer subgroups from one stage group to another (table 1). This was because differences in prognosis that held true in multiple populations suggested these were good places to draw lines between TNM descriptors and ways to combine subgroups together into stage groups. What has ensued, however, is a plethora of papers reporting the prognosis of an (often new) subgroup in a single population, suggesting further stage classification rearrangements. In addition, there are many papers identifying a new (unvalidated) prognostic factor and proposing that this be incorporated in an altered stage classification.

There is a lack of appreciation of the difference between the stage classification-which is meant to provide a nomenclature to describe the anatomical extent of a tumour-and a prognostic categorisation system - which can be used to estimate the prognosis. In part this stems from the fact that differences in prognosis were used as a tool to develop the stage classification, and that the anatomical extent of disease (ie, stage classification) is used as a tool in describing prognosis. But the use of one to help define the other should not obscure the fact that stage classification and prediction of prognosis are two different things.

The desire to predict prognosis is great. Certainly, the stage of a tumour is an important component influencing this. But

Correspondence to Dr Frank C Detterbeck, Yale University School of Medicine, 330 Cedar St, Thoracic Surgery, Yale University, New Haven, CT, 06520-8062, USA; frank.detterbeck@yale.edu there are many other factors such as performance status, histology, tumour genetic characteristics, prognostic factors, comorbidities - and perhaps most importantly - the treatment given (at least we like to believe it makes a difference).

The issue of wanting the stage nomenclature to provide us with an accurate prediction of prognosis is more acute with this edition of the stage classification than previously. We have more data and therefore have split stage classification into more subgroups. This is the information age, and we have an increasing wealth of data and are constantly developing more sophisticated ways to crunch the numbers to make them provide us with answers. All of this fosters the concept that, if only we include enough details, we will pinpoint the prognosis exactly.

Is an exact definition of prognosis actually achievable? In quantum physics the Heisenberg uncertainty principle states that certain pairs of physical properties, such as position and momentum, cannot be simultaneously known to an

arbitrarily high degree of precision-that is, the more precisely one property is measured, the less precisely the other can be measured. A similar phenomenon may be at work with regard to determining prognosis.

If we take only one aspect (ie, the anatomical extent of the tumour), we can amass large cohorts and report a prognosis with tight error bars. Of course this prognosis only applies to the conglomerate of patients included in the large cohort, which includes patients with many different oncological, genetic, medical, comorbid and socioeconomic characteristics that underwent various treatments of various qualities. The prognosis for any particular individual in the group is not likely to be the same as the average for the entire group.

On the other hand, if we parse out more and more subgroups according to each of these factors, we can arrive at a patient cohort that begins to approximate more closely the patient sitting in the clinic in front of us. However, as we add more and more data and split into more and more specific subgroups, we eventually end up with a 'group' containing a single patient, completely defined by all relevant factors. The problem at this level, of course, is that, even if we are able to come up with a number that characterises that person's individual prognosis, the Confidence Intervals around this number would range from 0 to $100 \%$ because of the size of the group. In other words, the more specifically we try to define prognosis, the less confident we can be that it is truly predictive. We can have either tight error bars for a large mixed conglomerate or wide error bars for a small very specific cohort.

We also live in an age where we tend to think we can influence everything. At the very least, we think that we should have been able to predict the economy, the

Table 1 Subgroups whose stage grouping assignment changed

\begin{tabular}{|c|c|c|}
\hline \multirow[b]{2}{*}{ General description } & \multicolumn{2}{|c|}{$\begin{array}{l}\text { Edition of stage } \\
\text { classification }\end{array}$} \\
\hline & 6th & 7th \\
\hline T2 ${ }_{5-7 \mathrm{~cm}}$ NO MO & $\mathrm{lb}$ & Ila \\
\hline $\mathrm{T}>7 \mathrm{~cm}$ NO MO & $\mathrm{lb}$ & Ilb \\
\hline $\mathrm{T}>7 \mathrm{~cm} \mathrm{N1} \mathrm{M0}$ & $\mathrm{llb}$ & Illa \\
\hline Malignant pleural involvement & Illb & IV \\
\hline $\mathrm{T} 2 \leq 5 \mathrm{~cm} N 1 \mathrm{MO}$ & llb & Ila \\
\hline Nodules same lobe NO MO & Illb & llb \\
\hline Nodules same lobe N1,2 M0 & Illb & Illa \\
\hline Ipsilateral lung nodules N0,1 M0 & IV & Illa \\
\hline Ipsilateral lung nodules N2,3 M0 & IV & Illb \\
\hline $\mathrm{T} 4{ }_{\text {invasion }} \mathrm{N0,1} \mathrm{MO}$ & Illb & Illa \\
\hline
\end{tabular}


weather and a patient's prognosis. Furthermore, if we know the prognosis, we immediately set out to intervene and change it: if it is bad we need to add additional treatment. In other words, as soon as we believe we have defined the prognosis, we set out to undermine our system of identifying it. We tend to forget that all our statistics and data only provide an estimate of prognosis, and we often forget to ask whether the treatment we are giving actually makes a difference. We forget to ask to what extent outcomes are actually explained by all of the information we have, and to what extent it is due to unknown factors (should we call this random events, fate or the will of God?). We tend to forget this because, when we do evaluate it, we find that the known factors explain only a small amount of the actual outcomes.
Perhaps it is time for us to step back, relax and think more carefully. We should let stage classification be a nomenclature to describe the anatomical extent of the disease and not ask more of this system. We should continue to investigate prognostic factors and try to come up with ways to deal with the complexity of the many factors and domains involved (tumour-related, genetic, patientrelated, treatment-related, socioeconomic, geographically-related, etc), and the fact that a factor may be prognostic only within a particular subgroup. But we should be patient and allow this process to mature. The demands for an accurate prognostic categorisation system are too simplistic and unrealistic. Furthermore, we should recognise that any system can at best only provide a partial estimate of prognosis. We should use science to our advantage wherever we can, but at the same time maintain an appreciation for the fact that some things cannot be determined precisely. Finally, no amount of more and more detailed data is ever going to remove all the complexity and yield a single simple system that provides a nomenclature, accurately predicts prognosis and serves as a treatment algorithm that obviates the need to think carefully about both the evidence and uncertainties that exist.

\section{Competing interests None.}

Provenance and peer review Not commissioned; externally peer reviewed.

Received 18 March 2011

Accepted 5 May 2011

Published Online First 27 May 2011

Thorax 2011;66:1016-1017.

doi:10.1136/thoraxjnl-2011-200214 